\section{Tomato seed saponins promise to cure dermatitis}

Dr Hiroshi Shimoda from Oryza Oil \& Fat Chemical Co Ltd and Kinda Univerity conduated a study on the conducted composition and bioactive properties of tomato seeds. The researchers found steroidal saponins called lycoperosides, previously associated with skin-health promotion, to be the major seed components, more abundant in seeds than in tomato fruits. The researchers that these saponins cure

inflammation and damaged skin in atopic dermatitis.

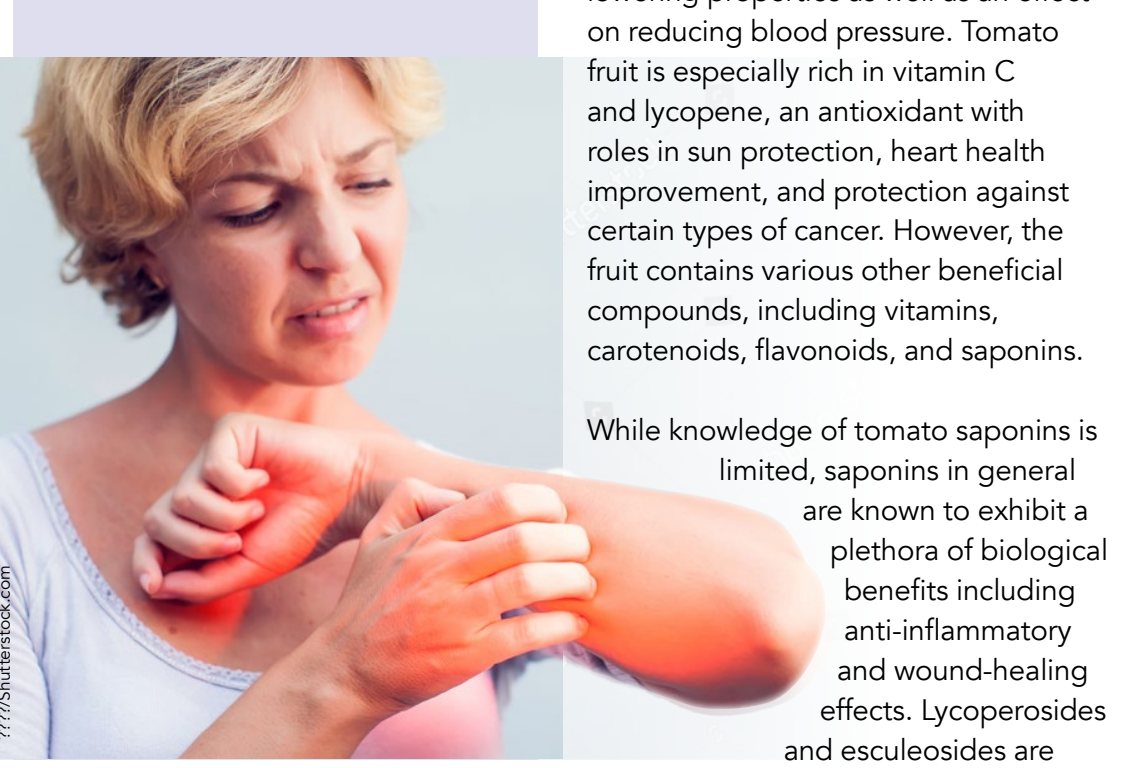

r Hiroshi Shimoda's laboratory is
dedicated to the study of edible seed components. One example of these components are molecules flavonoids that protect seed oils from oxidation. These types of molecules are beneficial to our health, which is why we use the seed extracts that contain them to produce dietary supplements, as well as Food for Specified Health Uses, also known as FOSHU products. The recent focus of Shimoda's studies has been on saponins found in tomato seeds.

\section{TOMATOES, SAPONIN}

AND DERMATITIS

Tomatoes originated in the Andes highlands in South America, but today they are grown worldwide. Tomato fr can be consumed raw or processed lorting properties as well as an effect is especially rich in vitamin $C$ with

政 exhibit fects. Lycoperosides and esculeosides are steroidal saponins that have been A a mer fof thes. Esculeoside of saponin compound found in fully ripe tomato fruits, was reported to prevent arteriosclerosis in mice. It also reported that orally administered esculeoside A reduced serum cholesterol in mice deficient in lipidbinding proteins. Moreover, it was found that oral administration of esculeoside $B$ isolated from tomato juice improved type IV allergic dermatitis in mice.

Dermatitis is a broad term that describes a common skin irritation. It has a number of causes and many forms which usually involve a rash, or itchy the drin might As result of dermatitis, the skin might blister, ooze, crust, or alleric dematis with complex and diverse symptoms. The pathology of $A D$ is characterised by pruritur (itchy skin), severe inflammation, and keratosis (a noncancerous skin condition manifested as a waxy brown, black, or tan growth). $A D$ can be treated by the systemic application of corticosteroids of varying strength, as well as by using an immunosuppressive ointment, such as tacrolimus.

\section{SAPONINS FOR HEALTHY SKIN} Saponins are generally recognised to have a smoothing effect on our skin when applied topically. Some saponins can also promote wound healing and There are many when applied orally. in the literature exhibiting these effects.

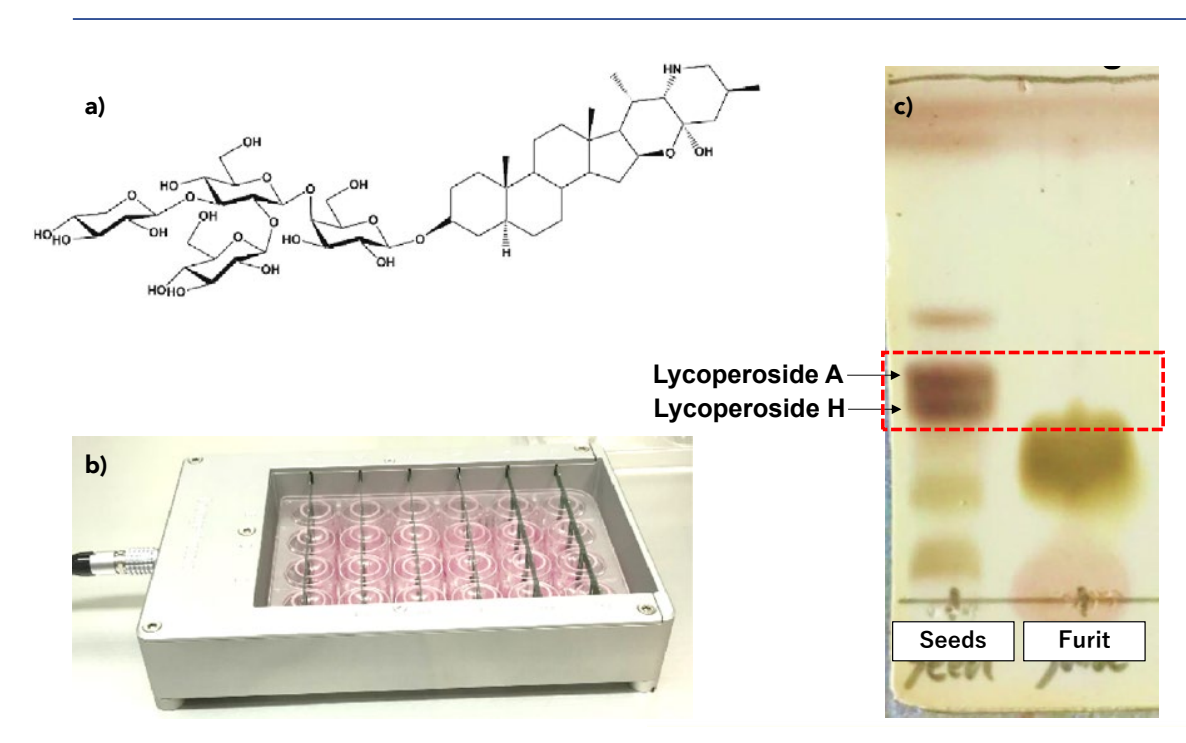

a) Structure of $L$ H, b) A device to measure TEWL in $3 D$ cultured cells, c) Saponin contents in
tomatoes $T \perp C$ chromatogram.

One example are centella saponins, found in the leaves and stems of the which are known for promoting collagen production and stimulating wound

healing Another widely known example

is Korean ginseng which contains a

type of saponin named ginsenosides.

formulation comprised of $C$. asiatica moisturisation and antin and anti-inflammation.

\section{FROM THE SEED TO}

THE MOUSE TO THE CURE

While much is known about the

It was found that

of ginsenoside-

containing Korean

ginseng extract

improved trans

loss (TEWL), which

reflects skin barrier

function in mice with $\mathrm{AD}$ indicating an

increase in anti-inflammatory effects

and skin hydration. Ginsenoside Rg 1;

in particular, mitigated the increase

damaged by UV radiation.

Apart from anti-inflammatory activity, ginsenoside Rc contributed to

epidermal moisture retention throug upregulation of key genes involved in the process. TEWL in AD mouse models was also improved by gracillin, a spirostan-type saponin isolated from the plant Dioscorea quinqueloba. Saponins from the plant Centella asiatica were found to reduce epidermal dehydration as well. Specifically, a major pentacyclic as werpene saponin called madecassoside, present in $\mathrm{C}$. asiatica, reduced TEWL and enhanced several moisturising molecules, including aquaporing, loricin, and involucin nd bioactive activity of tomato see and found lycoperoside saponins to abundant in seeds than in tomato fruits. Based on the reported effects of saponins, Shimoda and associates hypothesised that saponins from tomato seeds may cure inflammation and damaged skin in $A D$.

Until recently, NC/Nga inbred mice have been used as rodent models in AD research aimed at identifying viable drug candidates, including natural

products. However, to induce symptoms of edema or swelling caused by excess fluid trapped in the tissues, a particullor chemical would have to be topically applied to the ears of these mice. Nowadays, in terloukn (L) 33 transgenic (LL-33Tg) mice that do not require any aternative to NCINga mice to as ans the efficacy of $A D$ remedies.

Loosely defined, cytokines are strong bioactive proteins that are important in cell signalling and IL-33 is a cytokine Shimoda and Morikawa investigated role in allergic the effects of tomato seed extract and lycoperoside $\mathbf{H}$ on atopic $\quad$ and it serves as an signal that activates innate immunity as it is released rapidly after cellular $\begin{array}{ll}\text { seed was lacking. Shimoda and his } & \text { damage or stress. In IL-33Tg mice, } \\ \text { colleague Professor Toshio Morikawa } & \text { IL-33 is upregulated which leads to } \\ \text { investigated the chemical composition } & \text { AD-like inflammation. The transgenic }\end{array}$ $\begin{array}{ll}\text { seed was lacking. Shimoda and his } & \text { damage or stress. In IL-33Tg mice, } \\ \text { colleague Professor Toshio Morikawa } & \text { IL-33 is upregulated which leads to } \\ \text { investigated the chemical composition } & \text { AD-like inflammation. The transgenic }\end{array}$ investigated the chemical composition
animals exhibit epidermal hyperplasia,

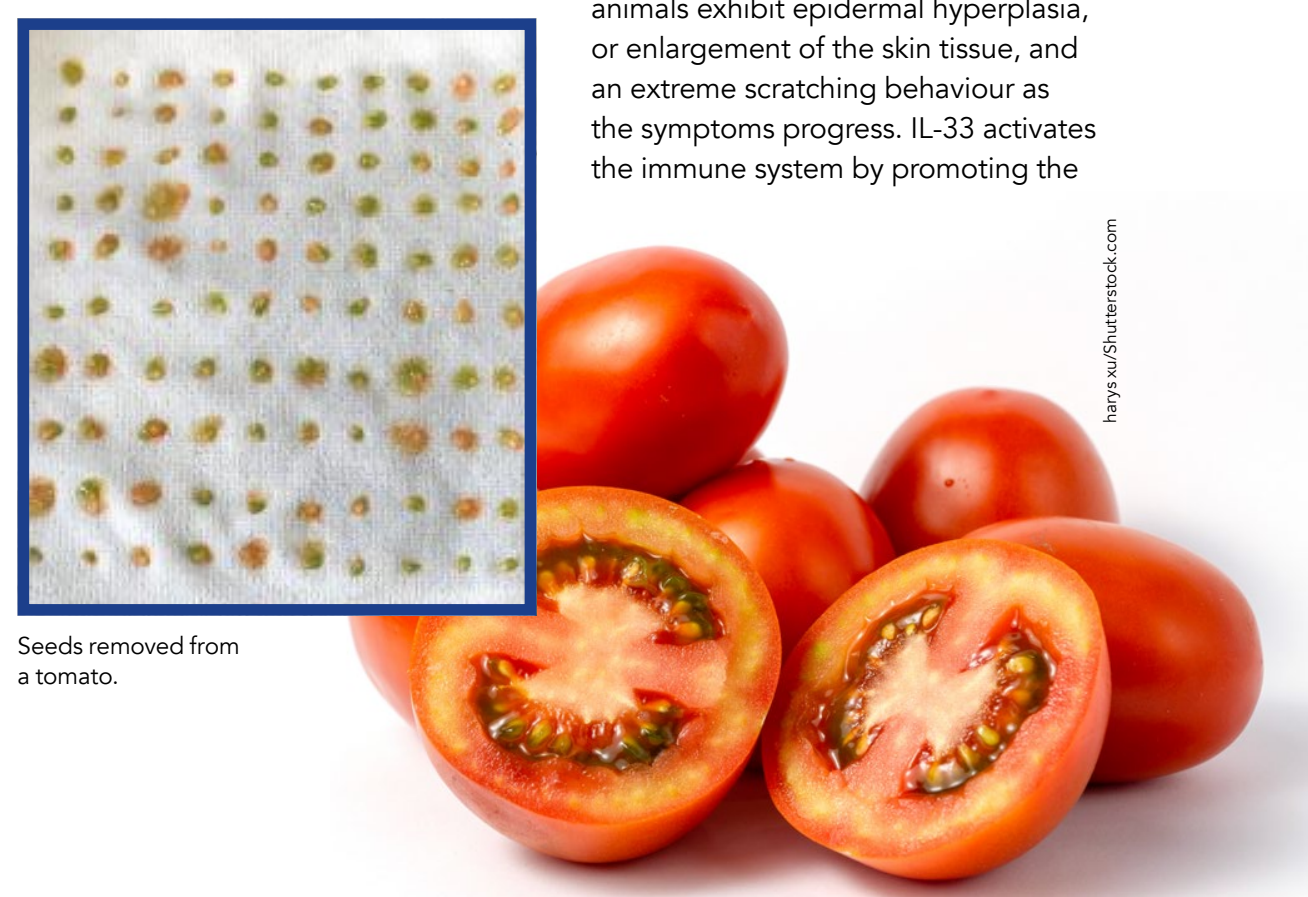


activation of Th2 lymphocytes, mast cells, and eosinophils. Consequently, cytokines, which hocytes release $-9,-10$, and -13 . Of these cytokines, $1-4$ and -13 have been associnted wit the deterioration of $A D$ symptoms. Therefore, these cytokines are studied

\section{TOMATO SEED}

TO CURE DERMATITIS

In light of previous findings that

tomato saponins had a suppressive

effect on AD, Shimoda and associates investigated the effects of tomato

seed extract (TSE) and lycoperoside

$H$ (LH) on AD symptoms in IL-33T mice. This was the first study to demonstrate the efficacy of natura products in this mouse model. In their study, the scientists gave TSE or result of four-month administration AD inflammation and TEWL were suppressed by TSE and LH. TSE slightly suppressed scratching behaviour while TSE and LH both increased locomotive activities. Moreover. LH administration clearly suppressed the accumulation of inflammatory cells in skin lesions and epidermal hypertrophy. Therefore,
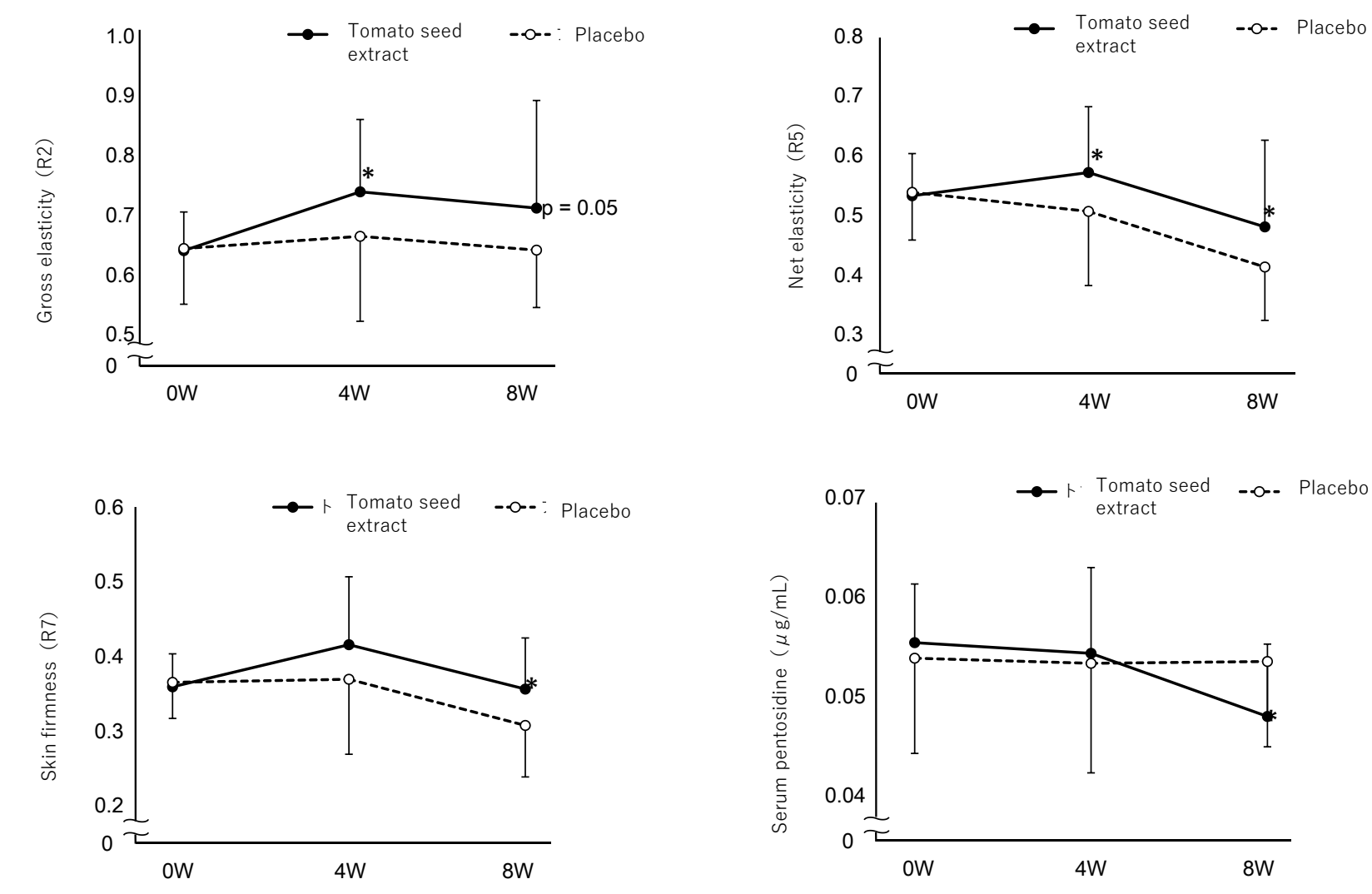

further investigate other saponins from tomato seeds, Shimoda and Morikawa custured apidermis model, alm a tested tomato seed saponins reduced additionally beneficial as it was the only compound found to boost skinbarrier function through increased ceramide production. $\begin{array}{ll}\text { are often used as dietary supplements } & \text { TSE containing } L H \text { has been } \\ \text { and beverages to aid skin hydration. } & \text { commercially available since } 2019 \\ \text { Another way of improving dry skin } & \text { it has been marketed as being able } \\ \text { symptoms is to use compounds that } & \end{array}$ $\begin{array}{ll}\text { are often used as dietary supplements } & \text { TSE containing LH has been } \\ \text { and beverages to aid skin hydration. } & \text { commercially available since } 2019 \text { and } \\ \text { Another way of improving dry skin } & \text { it has been marketed as being able } \\ \text { symptoms is to use compounds that } & \end{array}$ symptoms is to use compounds that

While much is known about the composition and properties of tomato fruit, similar knowledge on the tomato seed was lacking.

can increase skin ceramide by inducing to enhance skin elasticity. This role moisturising crempared to applying the body. of TSE and $L \mathrm{H}$ was deduced while investigating barrier functions. Shimoda and associates are planning a new of $L H$ on reducing dry skin symptoms and they seek to further expand the To gain insight into the mechanism by knowledge base on the roles of tomato Wich LH inhibited AD symptoms and to seed saponins and LH. TEWL. However, $H$ was considered internal ceramide synthesis, Oral supplements are generally considered

\section{Behind the Research}

Dr Hiroshi

Shimoda

E: info@oryza.co.jp T: +81586865141

\section{Research Objectives}

Dr Hiroshi Shimoda and his team research tomato seeds as a potential cure for atopic dermatitis.

\section{Detail}

\section{Address}

Dr Hiroshi Shimoda, PhD

Oryza Oil \& Fat Chemical Co Ltd, Numata 1, Kitagata-cho, Ichinomiya, Aichi 493-8001, Japan

Professor Toshio Morikawa, PhD

Pharmaceutical Research and Technology Institute, Kindai Universt

Bio

Hiroshi Shimoda graduated from Kyoto Pharmaceutical University in 1990 and entered a pharmaceutical company. In 2000, he obtained a PhD (pharmaceuticall) at Osaka University, and in 2003 he joined Oryza Oil \& Fat Chemica Co. Since 2004 he has been Research and Development

Toshio Morikawa received his $\mathrm{PhD}$ from Kyoto

Pharmaceutical University in 2002. In 2001, he became an assistant professor in the same university. In 2005, he became a lecturer at Kindai University and was promoted to professor in 2015. His research interests involve searching for bio-functional molecules from

\section{Funding}

sidies for creative companies in Aichi prefecture.

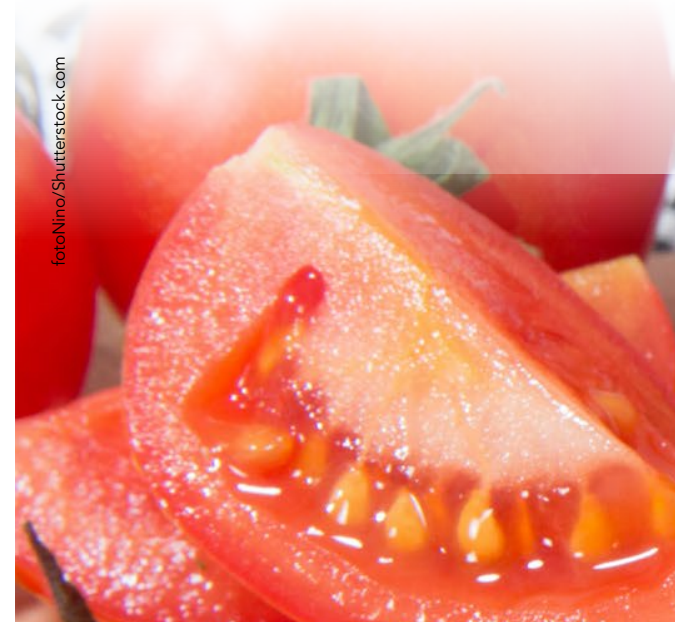

Professor Toshio Morikawa

E: morikawa@kindai.ac.jp T: +81643074306

\section{References}

Takeda, S, Miyasaka, K, Shimoda, H, (2021) Lycoperoside H, a steroidal alkaloid saponin in tomato seeds, ameliorates atopic dermatitis-llke symptoms in IL-33 transgenic mice. Jounnal of

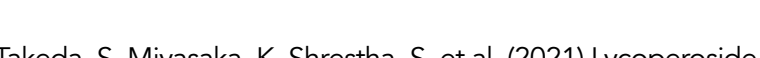
$\mathrm{H}$ a tomato seed saponin, improves epidermal dehydration reasing ceramide in the stratum corneum and steroidal anti-inflammatory effect. Molecules, 26(19), 5860. doi org/10.3390/molecules26195860

Oryza Oil \& Fat Chemical Co, Ltd, (2020) Tomato seed extract. Ver 2. www. oryza.co.jp/cms/wp-content/

\section{Personal Response}

\section{What advantages would $L H$ offer in treating $A D$} compared to the remedies that are already available? II One advantage of $L \mathrm{H}$ is cost. The price of $L \mathrm{H}(10 \mathrm{mg})$ or fewer than an Olumiant tablet (Lilly), a JAK inhibitor recently permitted as an orally effective $A D$ medicine in Japan. It is able to maintain a less inflammatory and hydrated skin condition for daily use. Another advantage is who have different ethnic and metabolic backgro of people No side effects have been reported so far Therefore appears to be a vegetable-derived safe $A D$ treatment. II

\section{Oryza}

ORYZA OIL \& FAT CHEMICAL CO., LTD. 